\title{
Reconstrucción de pene luego de necrosis de glande por priapismo
}

\section{Penile Reconstruction after Glans Necrosis by Priapism}

\author{
Federico Gaviria Gil ${ }^{1}$ Johana Andrea Benavides ${ }^{2}$ Adrián Lopera ${ }^{1}$ \\ ${ }^{1}$ Departmento de Urología, Hospital Pablo Tobón Uribe, Medellín, \\ Antioquia, Colombia \\ 2 Residente de Urología, Universidad CES, Medellín, Antioquia, \\ Colombia

\begin{abstract}
Address for correspondence Johana Andrea Benavides Martinez, Residente de Urología, Universidad CES, Medellín, Antioquia, Colombia (e-mail: johanainte08@hotmail.com).
\end{abstract}

Urol Colomb 2019;28:76-79.

\section{Resumen}

Palabras Clave

- priapismo

- necrosis de pene

- veno-oclusivo

- priapismo de bajo flujo
Introducción El priapismo se define como una erección parcial o completa que dura más de 4 horas en ausencia de estímulo sexual. Se clasifica en isquémico, no isquémico e intermitente. El primero corresponde a la mayoría de casos y puede producirse por un estímulo sexual o por medicamentos. El manejo inicial consiste en la aspiración de los cuerpos cavernosos y la inyección de simpaticomiméticos. Cuando esas medidas fallan, el manejo quirúrgico está indicado. Entre las complicaciones más frecuentes está la disfunción eréctil, sin embargo, en la literatura, hay muy pocos reportes de necrosis de pene.

Descripción del Caso Clínico Paciente de 60 años de edad que presentó priapismo isquémico luego de la inyección intracavernosa de prostaglandina E. Viene a consulta y le realizan lavado de cuerpos cavernosos con inyección de simpaticomiméticos que no fue exitoso; por lo que le realizan fístula espongio-cavernosa distal (Al-Ghorab) y rafia de uretra por lesión advertida. Consulta nuevamente por persistencia de edema, áreas necróticas y sangrado en glande. Se decidió iniciar manejo médico. Presentó evolución estacionaria y aumento en necrosis por lo que se derivó con cistostomía y se realizó desbridamiento de tejido necrótico inicialmente. Posteriormente se realizó Reconstrucción peneana + Uretroplastia peneana + Lavado desbridamiento de tejidos + Cistoscopia con buena evolución clínica.

Conclusiones Existen diferentes factores de riesgo para presentar priapismo de origen isquémico como es la inyección de medicamentos intracavernosos. La principal complicación que se presenta es la disfunción eréctil. Sin embargo, hay pocos casos descritos de necrosis de glande. En el caso de nuestro paciente se plantean diferentes hipótesis como factores contribuyentes a la necrosis como son el uso de simpaticomiméticos y/o la desvascularización del glande posterior a la cirugía. Al enfocar ese tipo de paciente, es indispensable realizar un adecuado manejo inicial para prevenir ese tipo de complicación que, aunque es poco frecuente, puede resultar catastrófica. received

October 8, 2017

accepted

March 10, 2018

published online

May 2, 2018
DOI https://doi.org/

$10.1055 / \mathrm{s}-0038-1648236$.

ISSN 0120-789X.

eISSN 2027-0119.
Copyright (c) 2019, Sociedad Colombiana License terms de Urología. Publicado por Thieme Revinter Publicações Ltda., Rio de Janeiro, Brazil. Todos los derechos reservados. 


\begin{abstract}
Keywords

- priapism

- glans necrosis

- veno-oclusive

- low-flow priapism

Introduction Priapism is defined as a prolonged and persistent penile erection that is unrelated to sexual stimulation and lasts longer than 4 hours in duration. It is classified as ischemic (low flow), non-ischemic (high flow), and stuttering (recurrent). Ischemic priapism is the most frequent. Initial treatment consists of corpora cavernosa aspiration. When it fails, surgical treatment is indicated. Erectile dysfunction is a frequent complication, however glans necrosis has been reported too.

Case Report A 60 year-old patient with ischemic priapism after prostaglandin E intracavernous injection consulted. Initial treatment was corpora cavernosa aspiration without detumecense and then a distal espongio-cavernous fistula was done. Some days later, he presented edema, bloods and necrotic areas in his glands. Conservative medical treatment was done without success and a surgical treatment was considered. Reconstructive procedure was done with good evolution.

Conclusions Ischemic priapism could be presented after intracavernous injection with low frequency. However, an appropriate treatment has to be done to prevent complications as penile necrosis. In this patient, there are some risk factors that could be contribute to glans necrosis as simpatico-mimetics used, lost of vascularization with surgery. When focusing on this type of patient, it is essential to carry out an adequate initial management to prevent this type of complication that, although rare, can result catastrophic.
\end{abstract}

\section{Introducción y Objetivos}

El priapismo se define como una erección parcial o completa que dura más de 4 horas en ausencia de estímulo sexual. ${ }^{1}$ Se produce por una disfunción de los mecanismos que regulan la tumecescia, rigidez y flacidez del pene. ${ }^{2}$ Se clasifica en bajo flujo (veno-oclusivo), alto flujo (arterial) e intermitente. ${ }^{3}$

El priapismo isquémico (veno-oclusivo) corresponde a la mayoría de los casos reportados en la literatura y es en gran parte, desencadenado por un estímulo sexual o por medicamentos. ${ }^{4}$ Existe un gran listado de medicamentos que pueden generar priapismo; pero entre los más frecuentes mencionados están las prostaglandinas intracavernosas con una incidencia que varía entre el 1\%-5.3\%. ${ }^{5}$

El manejo está enfocado en recuperar la flacidez, disminuir el dolor y evitar el daño crónico en los cuerpos cavernosos. ${ }^{6}$ El tratamiento inicial consiste en la descompresión de los cuerpos cavernosos con aspiración; y sino mejora, se emplea la inyección de medicamentos simpaticomiméticos como la epinefrina y la fenilefrina. ${ }^{7}$ Cuando esas medidas fallan, el manejo quirúrgico está indicado. ${ }^{6,8}$

Entre las complicaciones más frecuentes está descrita la disfunción eréctil. Sin embargo, en la literatura hay muy pocos reportes de necrosis de pene. ${ }^{9}$

\section{Descripción del Caso}

Paciente de 60 años sin antecedentes de importancia a quien en estudio para disfunción eréctil le realizaron una ecografía más doppler peneano, con inyección intracavernosa de prostaglandina E2 posterior a lo cual presentó priapismo de bajo flujo.

Consulto inicialmente y fue sometido a lavado de cuerpos cavernosos más inyección de simpaticomiméticos (epinefrina) con persistencia del priapismo; razón por la cual le realizan fístula espongio-cavernosa distal con técnica de Al-Ghorab y rafia de la uretra por lesión advertida con cromado 3/0. Estuvo hospitalizado por 2 semanas y dieron egreso.

Posteriormente, consultó al servicio de urgencias de la institución por persistencia de edema y sangrado en el surco balano-prepucial.

Al examen físico presentaba uretrorragia, algunas áreas necróticas en la piel del glande, pero la mayor parte con adecuada vitalidad. Los cuerpos cavernosos se palpaban fibróticos y la sutura en el surco balano-prepucial se veía sana (-Fig. 1). Al paso de la sonda vesical tenia salida de lidocaína en la parte lateral izquierda del surco balanoprepucial.

Se decidió en ese momento, iniciar cubrimiento antibiótico y continuar curaciones por clínica de heridas. Sin embargo, el paciente presentó evolución estacionaria con aumento en la necrosis y además una fístula uretro-cutanea, por lo que se derivó con cistostomia y se realizó desbridamiento del tejido necrótico (-Fig. 2).

El paciente continuó las curaciones por clínica de heridas. Veinte y seis días después; una vez los tejidos estuvieron sanos y se observaba tejido de granulación; se realizó reconstrucción peneana más uretroplastia, más lavado y desbridamiento de tejidos con adecuada evolución clínica.

\section{Descripción de la Técnica Quirúrgica}

En los hallazgos durante el procedimiento, presentaba necrosis del pene a nivel dorsal, con defecto en el glande, el surco balanoprepucial y el cuerpo distal del pene de $3 \times 2 \mathrm{~cm}$. Además, tenía una lesión de la uretra de $1.5 \mathrm{~cm}$ y un defecto de $1 \mathrm{~cm}$ en cada uno de los cuerpos cavernosos (-Fig. $\mathbf{3}$ ).

En cirugía lo que se hizo inicialmente fue desbridar el tejido necrótico y con fibrina. Se liberaron los bordes de la túnica 


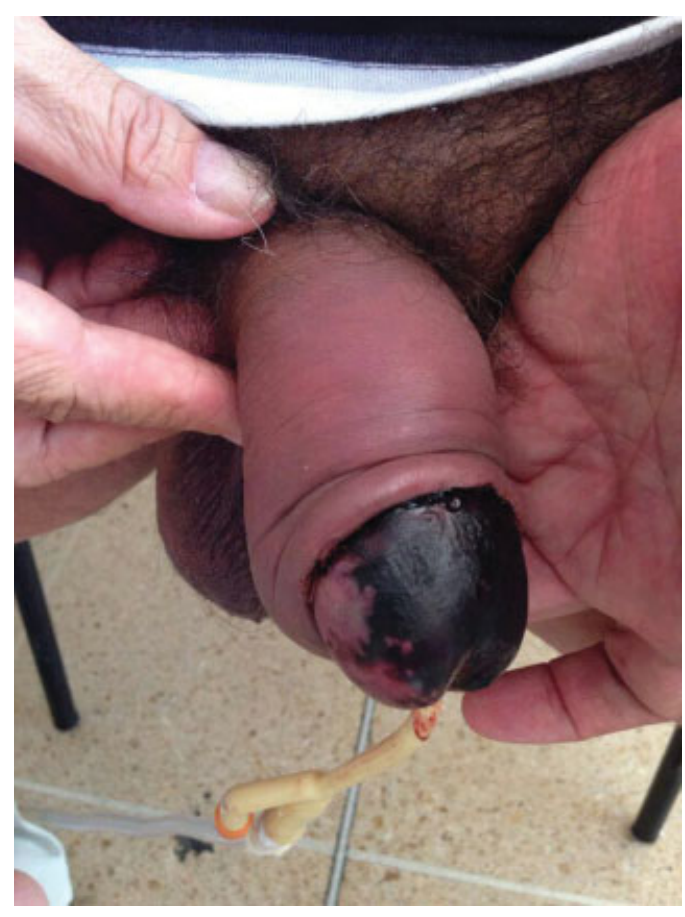

Fig. 1 Áreas necróticas en piel de glande. Sutura en surco balanoprepucial sana.

albugínea y se realizó la rafia de los cuerpos cavernosos con sutura continua con vicryl $3 / 0$. Se calibró la uretra con bugía de Hegar \# 7 y se realizó el cierre dorsal de la mucosa uretral con 6 puntos separados con vicryl 4/0 libres de tensión. Se pasó una sonda Foley de silicona 14Fr. Posteriormente, se avivaron los

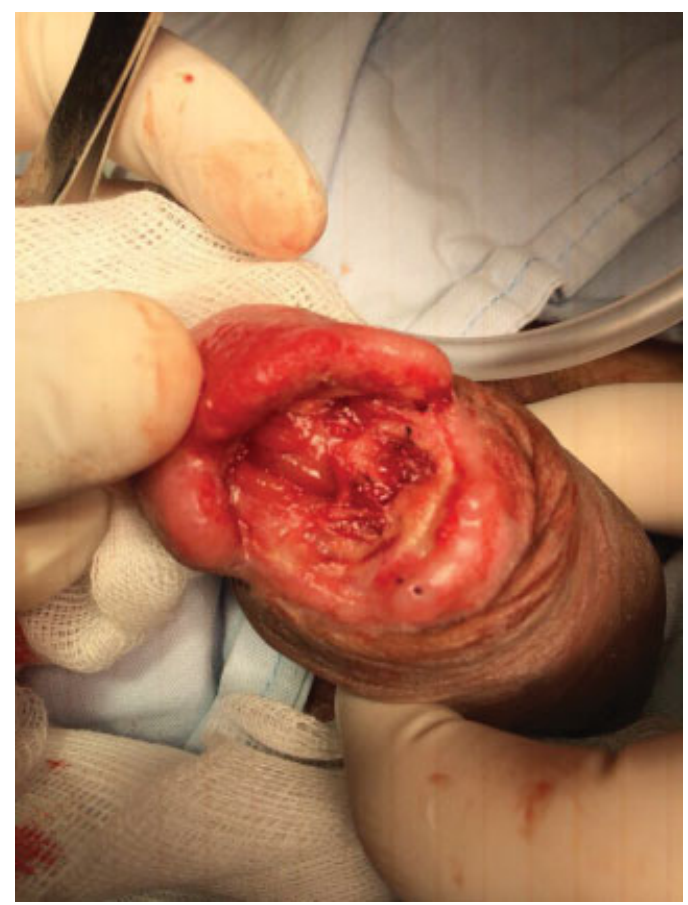

Fig. 2 Presentaba desprendimiento del glande en un $80 \%$ con áreas de necrosis en surco balano-prepucial del lado izquierdo, lesión de uretra en su parte distal y apertura del cuerpo cavernoso izquierdo Se realizó lavado más desbridamiento de tejido necrosado y se fijó el glande al cuerpo cavernoso con punto de vicryl 3/0.

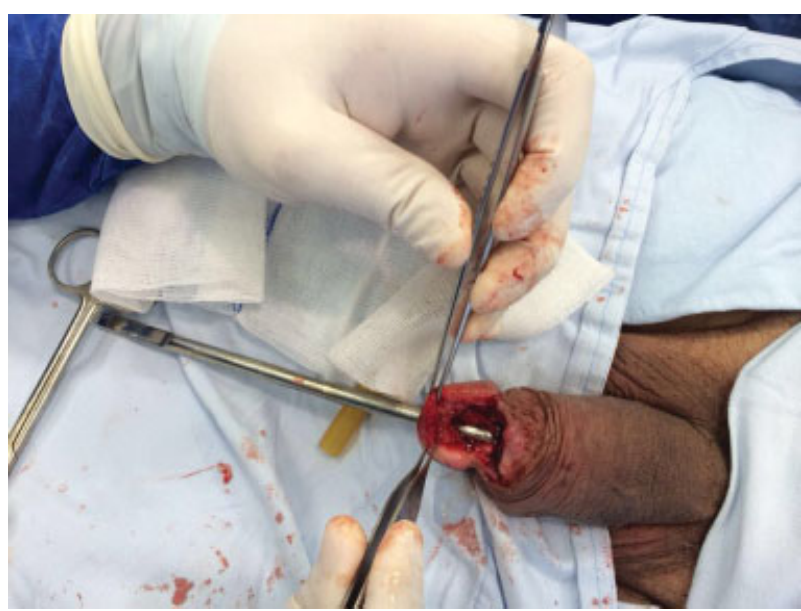

Fig. 3 Defecto de tejido en el glande, surco balano prepucial y cuerpo distal del pene de $3 \times 2 \mathrm{~cm}$ con lesión profunda que compromete la pared dorsal de la uretra. Lesión de uretra de $1.5 \mathrm{~cm}$, lesión de cuerpos cavernosos a nivel distal con defecto de $1 \mathrm{~cm}$ en cada uno. No se detectaron signos de infección, se palpaban ambos cuerpos cavernosos duros hasta la base del pene por fibrosis.

bordes de la piel del glande en el sitio del defecto y se pusieron puntos hemostáticos de afrontamiento del glande por planos con vicryl 3/0 (-Fig. 4). A las 3 semanas se le retiró la sonda vesical y la cistostomia con adecuado flujo miccional.

\section{Discusión}

Se describe el caso de un paciente que presentó necrosis de pene posterior a un priapismo inducido por la inyección

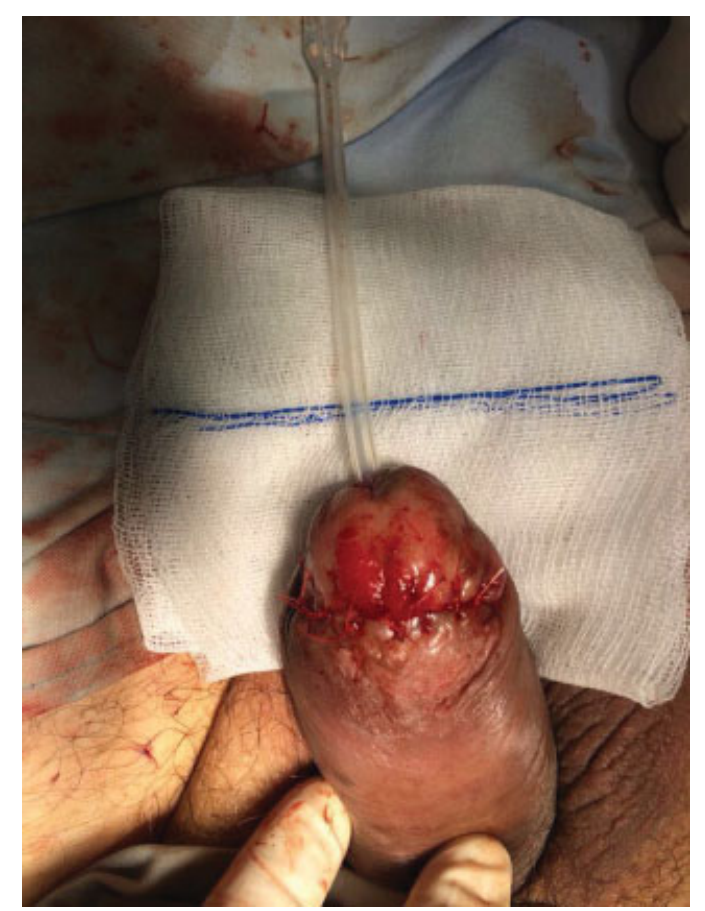

Fig. 4 Se realizó uretroplastia con cierre dorsal de la mucosa con vicryl 4-0 con 6 puntos separados libres de tensión previo al paso de sonda Foley de silicona 14 fr. Puntos hemostáticos de afrontamiento del glande por planos con vicryl 3-0. 
intracavernosa de prostaglandina E2, con mala respuesta al manejo inicial por lo que requirió manejo quirúrgico presentando necrosis de pene y asociado a lesión advertida de uretra.

En la literatura, hay pocos casos reportados de necrosis de pene, la serie más grande está descrita por Martinez ${ }^{9}$ donde reportan 18 casos de los cuales solo 2 fueron secundarios a priapismo. Parth Patel ${ }^{10}$ reporta el caso de un paciente que presentó gangrena de pene con formación de absceso posterior a la corrección con técnica quirúrgica de Al-Ghorab.

Todo eso, aunque muy poco frecuente, puede ocurrir a pesar de la rica irrigación vascular del pene, ${ }^{11,12}$ porque la vasculatura de los cuerpos cavernosos es susceptible de insuficiencia vascular en ese caso, por la posible ligadura de los vasos en cirugía, asociado al uso de medicamentos simpático miméticos y el mismo priapismo. ${ }^{13}$

\section{Conclusiones}

El priapismo de origen isquémico se puede presentar posterior a la inyección de medicamentos intracavernosos con relativa frecuencia. El urólogo debe estar en capacidad de realizar un adecuado manejo inicial para evitar complicaciones.

En caso de presentarse, el adecuado abordaje y manejo es indispensable para poder evitar complicaciones más graves, como la necrosis de pene que fue la complicación que presentó el paciente.

En ese caso, el paciente no tenía comorbilidades que aumentaran el riesgo de esa complicación. Sin embargo, los factores que se sugieren como precipitantes fueron los simpaticomiméticos y posible vasculopatía. Es necesario realizar un estudio con una serie de casos más amplia para confirmar los factores de riesgo y poder prevenirlos.

\section{Responsabilidades Éticas}

Protección de personas y animales. Los autores declaran que para esta investigación no se han realizado experimentos en seres humanos ni en animales.

Confidencialidad de los datos. Los autores declaran que han seguido los protocolos de su centro de trabajo sobre la publicación de datos de pacientes.
Derecho a la privacidad y consentimiento informado. Los autores declaran que en este artículo no aparecen datos de pacientes.

Conflictos De Interés

No existen.

Financiación

Recurso logístico y humano propios.

\section{Bibliografía}

1 Burnett AL, Bivalacqua TJ. Priapism: current principles and practice. Urol Clin North Am 2007;34(04):631-642, viii

2 Shigehara K, Namiki M. Clinical Management of Priapism: A Review. World J Mens Health 2016;34(01):1-8

3 Ridgley J, Raison N, Sheikh MI, Dasgupta P, Khan MS, Ahmed K. Ischaemic priapism: A clinical review. Turk J Urol 2017;43(01):1-8

4 Hudnall $M$, Reed-Maldonado $A B$, Lue TF. Advances in the understanding of priapism. Transl Androl Urol 2017;6(02):199-206

5 Costa P, Potempa A-J. Intraurethral alprostadil for erectile dysfunction: a review of the literature. Drugs 2012;72(17):2243-2254

6 Salonia A, Eardley I, Giuliano F, et al; European Association of Urology. European Association of Urology guidelines on priapism. Eur Urol 2014;65(02):480-489

7 Falcone M, Gillo A, Capece M, Raheem A, Ralph D, Garaffa G. The management of the acute ischemic priapism: A state of the art review. Actas Urol Esp 2017;41(10):607-613

8 Pal DK, Biswal DK, Ghosh B. Outcome and erectile function following treatment of priapism: An institutional experience. Urol Ann 2016;8(01):46-50

9 Montoya Martínez G, Otero García JM, López Samano V, González Martínez J, Serrano Brambila E. [Penile necrosis: a review of 18 cases at the Hospital de Especialidades Centro Médico nacional Siglo XXI]. Arch Esp Urol 2006;59(06):571-576

10 Ford-Glanton BS, Patel P, Siddiqui S. Penile gangrene with abscess formation after modified Al-ghorab shunt for idiopathic ischemic priapism. Case Rep Urol 2014;2014:705417

11 Babaei AR, Safarinejad MR, Kolahi AA. Penile revascularization for erectile dysfunction: a systematic review and meta-analysis of effectiveness and complications. Urol J 2009;6(01):1-7

12 Benavides J, García H. Priapismo y anemia de células falciformes: una revisión de la literatura. Rev Urol Colomb 2013;22(02):37-42

13 Chiang I-N, Chang S-J, Kuo Y-C, Liu S-P, Yu H-J, Hsieh J-T. Management of ischemic penile gangrene: prompt partial penectomy and other treatment options. J Sex Med 2008;5(11):2725-2733 